\title{
Japanese Honorifics and Situation Semantics
}

\section{R.Sugimura}

Institute for New Generation Computer Technology(ICOT) Japan.

\section{ABSTIRAC'I}

A model of Japanese honorific expressions in situation semantics is proposed. Situation semantics provides considerable power for analyzing the complicated structure of Japanese honorific expressions. 'The main feature of this model is a set of basic rules for context switching in honorific sentences. Mizutani's theory of Japanese honorifics is presented and incorporated in the model which has been used to develope an experimental system capable of analyring honorific context. Some features of this system are described.

\section{Introduction}

The Japanose language, like Korean and many non-libropean languages, contains a grammaticalized system of honorific forms. It is well known that the use of honorifics is closely connected to context, including aspects like relative social standing. No effective mechanisms have been developed to deal with this problem. Situation Semantics (SS) lBarwise $1982,1984 a, 1984 b, 1985 a, 1985 b, 1985 c, 1985 d, 1985 c||$ Barwise \& Perry 1983] [I,experance][[Pollard 1985 ][Creary \& Pollard] is a theory of context used here to construct a model of honorifie sentenees to analyze the relationship between sontence and context

About Japanese, we can make use of Mizutani's theory of honorifies [Mizutani 1983a,1983b]. 'This theory does tackle the relation between context and sentence, but it seems that SS can describe context move usefully than Mizulini's theory. In this paper, SS is used to reconstruct the context given by Mizutani's theory. Honorific forms are analyzed and basic rules for context switching are established.

Table 1 gives the relation between Mizutani's theory of honorifies and the model. 'Ihis model can be divided into two parts. 'l'he first part rleseribes basic context features and the second describes lexical rules based on Mizutani's Japanese grammar, along with some basic mechanisms of "context switching."

'I'able 1

\begin{tabular}{|c|c|c|c|}
\hline Context of honorifics & $\begin{array}{c}\text { Mizutani's l'heory } \\
\text { as vector element }\end{array}$ & $\begin{array}{c}\text { Our Model } \\
\text { as event type in SS }\end{array}$ & 1st \\
\hline Discourse formalism & none & SS & part \\
\hline Grammar & $\begin{array}{c}\text { Japanese grammar } \\
\text { in ClG Form }\end{array}$ & $\begin{array}{c}\text { Japanese grammar } \\
\text { in DCG form }\end{array}$ & 2nd \\
\hline $\begin{array}{c}\text { Context switch in } \\
\text { honorific sentence }\end{array}$ & none & In complex sentence & part \\
\hline Implement & none & In CLL & \\
\hline
\end{tabular}

It is very casy to represent some context features in discourse in SS, but context involves some very difficult problems like "focusing." We want to analyze this in future research. In this paper only cnough elements for context required in Mizulani's theory are set up.

The main concern here is the second part of the model which deals with the relationship between contextual elements and lexical elements, and especially the mechanism of "context switching" on honorifics.

Mizutani's theory of honorifie expression in Japanese is described first Then the model in SS is presented, 'The conlext feature of relative social status in Mizutani's theory is realized in the first part of the model. 'l'his is followd by explanation of some basic features designed to handle the mechanism of conlioxt switching in the second part. Finally, an experimental system based on this model is given. 'I'his system was implemented in CH. (Complex Indeterminate Language)/Mukai 1985a, $1985 \mathrm{bl}$. Results from some experiments processing Japanese honorific expressions are given at the end of the paper.

\section{Mizutani's theory of Japanese honorific expression}

2.1. Honorific Status

Honorific relations are represented as vectors in an abstract two_dimensional homorific space. In the honorific space the speaker in tho discourse is set as the origin. Other individuals like hearer and agents who are presented in the sentence of discourse are represented by vectors as in the following example.

An honorific atlitude is defined as the vector between these points. For example, the honorific attitude from the speaker (I) to the hearer ( $Y$ ) is defined as a vector from $\mathrm{I}(0,0)$ to $\mathrm{Y}(\mathrm{y}, 1, \mathrm{y} 2)$. 'I'he honorific attitude from the

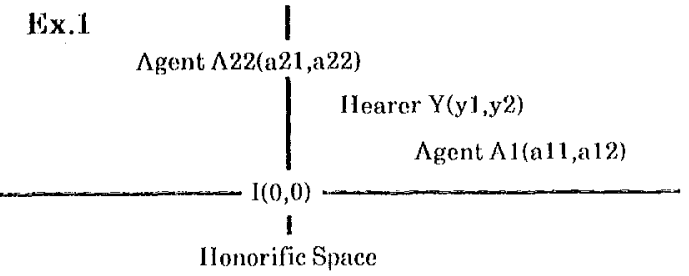

hearer $Y$ to agent2 is defined as the vector from $(y 1, y 2)$ to (a21, a22), i.e., the vector $(a 21-y 1, a 22-y 2)$

Next, we define the honorific value and the direction in the following way

Definition 1 Honorific value

F'or $t=\langle a 1, a 2\rangle$

honorific value of $\langle\mathrm{t}\rangle=$ a2 iff a1 $=0$;

Definition 2 Honorific direction

1) Up $\langle\mathrm{t}\rangle>0, \quad$ 3) lilat $\langle\mathrm{t}\rangle=0 \wedge \mathrm{a} 1=0$,

2) Jown $\langle\mathrm{t}\rangle\langle 0, \quad$ 4) $\Lambda$ cross $<t>=0 \wedge \mathrm{a} 1|=0$

We represent the "flat" honorific relation between agents explieitly, but the "across" relation is represented as in which there is no need to express the honorific relation explicitly. F'or conventional use, we clefine the following directions.

5) any1 up or down or flat, 6) any2 up or down or flat or across

To analyze the sentence utterod, definitions of the following honorific relations are neeessary.

Definition.3 Firstorder honorific relation

'T'he honorific attitude of the spealser to the hearer. $\overrightarrow{\mathrm{I} \mathrm{Y}}$

Definition 4 Second order honorific relation

'The honorific atlitude of the speaker' or the hearer toward the agent in the sentence of discourse. In this case the original point of the vector should be the point of the hearer or speaker, whichever is higher. If I $>$ II the original point of the vector will be 1 , and if $I<I l$ the origin will $I$. We introduce the notation $\mathrm{J}$ which stands for the higher individual.

$$
\overrightarrow{\mathrm{A}} \overrightarrow{\mathrm{s}}
$$

Definition 5 Third order honorific relation

'The honorific relation between agents of a discourse.

$$
\overrightarrow{A 1} \overrightarrow{\mathrm{A2}}
$$

\subsection{Japanese Gramma}

Now we can represent the relation between these three honorific relations and the sentences of discourse. Before looking at this honorific relation, we will show the small number of Japarese grammar rules used to define the structure of sample Japanese sentences. 'This grammar is taken from the "Sketch of Japanese Grammar" [Mizutani 1983al, a part of which is shown in Figure 1. 'lhis grammar is presented in $\mathrm{CF} G$, but we give the rules in IDCG

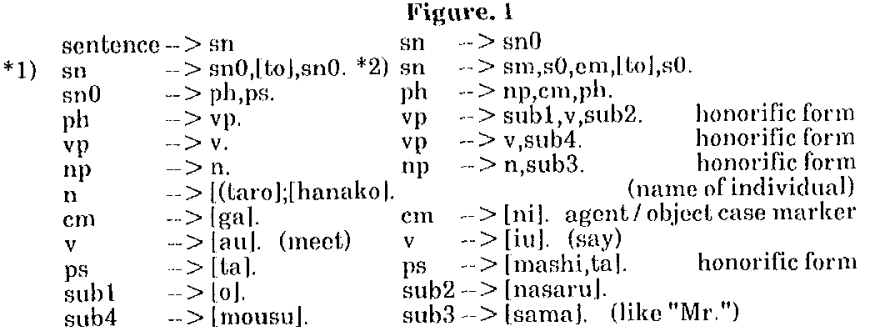

In his theory, Mizutani attaches Japanese terms to each terminal and non terminal node. 'T'able 2 vives the correspondence between Mizutani's Japanese terms and standard Winglish grammatical terms. Rules*1) and *2) above are not represented in his grammar. These rules were included specifically to represent direct and indirect speech 
Table. 2

sn(sentence nuclear $)=$ juttaiku cm(case marker) = kakuhyoji. ph (phrase element) $=$ jutsuso

$\operatorname{sn} 0($ sentence nuclear 0$)=$ juttaiku0 ps (post sentence marker) $=$ juttaiji $\mathrm{vp}$ (verb phrase) $=$ yorengo

$\mathrm{v}($ verb) $=$ doshi $\quad \mathrm{np}$ (noun phrase) - tairengo. n (noun)=taigen.

2.3. Helation between honorific context and Japanese grammar,

The following relation holds between honorific relations and the sentence of discourse. 'The notation $"==\geq$ " means the left element has some effect on the right element.

1) Changes in the the first order honorific relation are shown

$$
\begin{aligned}
& \text { Rule } 1 \text { relation } \\
& \qquad \mathrm{I}, \mathrm{H}===>\text { ps }
\end{aligned}
$$

2) Changes in the second order honorific relation are shown by description of individual $A 1$ and the honorific elements sub1 and sub2 of $v p$ in the agent case, and by description of individual $A 2$ as the np in the object case.

$$
\text { Rule } 2 \text { relation }
$$

$$
J, A 1===>\quad n p(\text { in agent case) } \quad \text { p (sub1 and sub2) }
$$$$
J, \Lambda 2===>\quad n p \text { (in object case) }
$$

3) 'The third order honorific relation is shown by $v$ in $v p$

Rule 3 relation

$$
A 1, A 2===>\quad v
$$

3. Model of the honorific sentence

In this section, we present our model in SS. Readers who are familiar with SS can skip Section 3.1.

3.1. First Part on Context Features

\subsubsection{Model of discourse}

In the theory of situation semantics, discourses are represented within situations and constraintis. In formal representation the sentence and context are expressed in the form "d,c [sentence]s, $e$ ". d stands for discourse situation (DU), $e$ is the speaker's connection (CS), $s$ is the setting and $e$ is the described situation. There is another situation called a resource situation in this theory. In our model we define a resource situation for each individual.

\section{(I) Discourse Situation (I)U)}

Here is an example of the representation of a sentence in discourse.

Ex 2. John said "l'om mot Jane" to Jim

$$
\text { el:= loc, } 10 \text {; yes }
$$

addressing John,Jim; yes uttering "Tom met Jane"; yes $10<1 d$ (ld means discourse location)

Discourse situations are represented in the following form as series of terms expressing relations between speaker $A$, hearer $B$ and uttered sentence [lalpha]]. In SS, spatio-temporal location is defined in discourse situations, but we have no effective way to formalize it in our model, so spatio-temporal location is not represented.

$$
\text { IU : = speaking } A \text {;yes addressing, } A, B \text {;yes saying,A,alpha;yes }
$$

(II) Speaker's Connection (CS)

The speaker's connection is a series of the following types. $A$ is the actual object and $[A]$ is the word that represents $A$.

CS:= speaking $A ;$ yes $\operatorname{refers} A[\Lambda]$; yes

\section{(III) Resource Situation(RS)}

A resource situation is defined for each individual in a discourse; it contains many events and constraints.

$\mathrm{RS}:=$ agent $A ; \mathrm{yes}$

$$
\begin{aligned}
& \text { has ; }\{\text { SOAs, } \\
& \text { Constraints Necessary,Nomic,Conventional } \\
& \text { Event type,., }\}
\end{aligned}
$$

3.1.2. Honorific context in the sentence

\section{(I) Honorific At titude Event type}

We introduce an "honorific attilude event type (Eh)" which stands for an honorific attitude in Mizutani's theory.

Formula 1) represents the mind status (HE) of the speaker towards Eh. A denotes the individual and "Ir" indicates that in the mind of the individual $A$, the honorific event type $\mathrm{Eh}$ is "represented." Eh shows the external honorific event including social honorifics.

Formulas 2) through 5) represent the basic honorific attitude event types (Eh). In Mizutani's theory, this event type is represented as a vector, but we represent it in the form of a binary relation. For one example, in 2) "honor-up" denotes the honorific relation between individuals $t$ and $t$ '. "ind $t \ldots$, yes" denotes that $t$ is an individual which can be represented in "_ "as its name.

1) $\mathrm{HE}:=\mathrm{Hr}, \mathrm{A}, \mathrm{Eh}$;yes

2) Eh: $:=$ honor_up t,t;yes

3) Eh: = honor_down t, t'; yes

4) $\mathrm{Eh}:=$ honor - across $\mathrm{t}, \mathrm{t}^{\prime}$;yes

5) $\mathrm{Eh}:=$ honor_eq $\mathrm{t}, \mathrm{t}^{\prime} ; \mathrm{yes}$

(II) Conditional Constraint on Word Selection

\section{Frame of Mind}

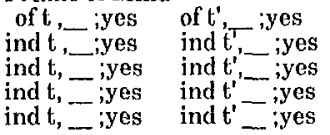

In Section 2.3 . we described the re grammatical rules. This section corresponds to Section 2.3 .
In Japanese there are many honorific words, and some interesting phenomena are to be found in daily life.

For example, a secretary in the company president's office should have many honorific words in his resource situation (RS) because he always has to be careful to use the appropriate honorific expression in his work. On the other hand university students will have a poor stock of honorific words for there is no need to express honorific status, except to teachers.

A constraint is required which determines the relation between the honorific event type ( $\mathrm{Fh}$ ) and word representation. This is the "conditiona constraint on word selection (Cw)." Mizutani's rules for first order through third order honorific relations given in 2.3. correspond to this constrain Cw. Word representation should be an event type called "honorific word selection event (IHW)", and if a person does not have this event type in his resource situation, he or she will have a poor range of honorifies. We represent these $\mathrm{CW}$ and $\mathrm{HW}$ in formula 6 )

In formula 6) "Cwl(Eh, 0 " denotes the conditional constraint of word selection Cw which has Eh and anchor "f" as its conditional schema. Anchor " $\mathrm{f}$ " determines the relation between indeterminates in $\mathrm{Eh}$ and objects like taro and hanako. See Barwise's work for details. 6) is read : if Eh is factual and $\mathrm{Cw}$ is salisfied, then $\mathrm{HW}$ is actual.

6) $\mathrm{Cw} \mid(\mathrm{Eh}, \mathrm{f}):=$ Involve Eh, HW ;yes

Conditional constraint on word selection has an honorific event type and its anchor as its scheme. In Japanese honorific expressions, if $\mathrm{Eh}$ and $\mathrm{f}$ are given, the word representation will follow very easily. Some instances of this are given in formulas 7) through 15). For example, formula 7) can be read : if $E$ h is actual and the anchor $f$ anchors $t$ to individual $J$ ( $J$ is the speaker or the hearer) or $t$ ' to $A 1$ in the agent case, then hw is actual so that when refering to $t$ ' honorific form tairen-go (np) is added to the description A 1

7) $\quad \operatorname{Eh}(f) f(t)=J, f\left(t^{\prime}\right)=A 1$ hw:= refer $t^{\prime},[\Lambda 1] \mid n p$ in h-form $]$

8) $\quad \operatorname{Lh}(\mathrm{f}) \mathrm{f}(\mathrm{t})=\mathrm{J}, \mathrm{f}\left(\mathrm{t}^{\prime}\right)=\Lambda 2$ hw: $=\operatorname{refer} \mathrm{t}^{\prime}$, (A2 $\| \mathrm{np}$ in h-form]

9) $\quad \mathrm{Eh}(\mathrm{f}) \mathrm{f}(\mathrm{t})=\mathrm{J}, \mathrm{f}\left(\mathrm{t}^{\prime}\right)=\mathrm{Al}$ hw:= refer $\mathrm{R},[\mathrm{R}][\mathrm{vp}$ in $\mathrm{h}$-form

10) $\quad \operatorname{Lh}(f) f(t)=A 1, f\left(t^{\prime}\right)=\Lambda 2$ hw: $=\operatorname{refer} R,[R \|$ vp in $h$-form

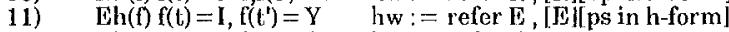

12) $\quad$ Wh $(f) f(t)=I, f\left(t^{\prime}\right)=\Lambda 1 \quad$ hw: = refer $t^{\prime},[t]$

13) $\quad \operatorname{Bh}(\mathrm{f}) \mathrm{f}(\mathrm{t})=\mathrm{l}, \mathrm{f}\left(\mathrm{t}^{\prime}\right)=\Lambda 2 \quad$ hw : = refer $\mathrm{t}^{\prime},[\mathrm{t}]$

3.2. Second Part on The Relation between Context Features and the Sentence

3.2.1. CIL as basic language

Before going into the representation of lexical rules which give the correspondence between honorific word expressions and sentences, and context switching on honorific sentences, we describe CIL used in the description of lexical rules. Accurate accounts of $\mathrm{ClL}$ can be found in [Mukai 1985a,1985b]. Here only the part needed to understand the lexical rules in section 3.2 .2 is described

CIL (Complex Indeterminate Language) can be represented by the following formula.

\section{CIL $=$ Prolog + Parameterized Types + Freeze}

\section{=Prolog + Frame +Freeze}

CIL has the unique data structure called "complex indeterminate." This data structure can be regarded as a frame and represented as in the following example.

(1) $\operatorname{term}((X$ with $a:=X, b:=Y$ where $X=Y))$.

(2) $>\quad \operatorname{term}(Z), a ! Z=a b c, b ! Z=a b c$.

yes.

Formula (1) is the specification of the data type and this can be used in formula (2). "a := $X$ " means assign value $X$ to slot $a . ~ " X=Y$ " denotes the condition part. If this condition is not satisfied the unification "term(X)" in (2) will fail. Formula (2) utilizes the complex indeterminate and unifies "abe" to each slot value.

3.2.2. DCG Rules for honorifics

(I) First order honorifics As Rule 1 in Section 2.3. shows, first order honorifics affect "ps." This is illustrated by the following examples.

Ex.3 This corresponds to $\mathrm{Eh}$ in 11 ) in section 3.1.2.

ps $(|\mathrm{ta}| \mathrm{X}], \mathrm{X}$, Contexl) $<$ -

dsolve(hono!((agent!(ds!Context))!(rs!Context) eq(speaker!(ds!Context),object!(lex!Context)))

"<-" denotes the operator ":-" in Prolog [Bowen 1982]. "[ta|X],X" is the DCG parsing mechanism. "Context" is a complex indeterminate variable for the context for this parsing part. "dsolve" is a Prolog predicate with the following mechanism.

dsolve $(X, Y)$. $X$ is a list like $[a, b, c, d]$ which contains Prolog atoms or terms. $Y$ is a Prolog atom or term.

1) Search list $X$ for $Y$

2) If there is a term in $X$ with the same arguments

but different term name, fail and return.

3) If $Y$ is not in $X$, then add $Y$ to list $X$, succeed and return.

4) If $Y$ is in $X$, succeed and return.

"hono!((agent!(ds!Context))!(rs!Context))" represents a list of honorific event types in the resource situation of the agent of discourse. "object! (lex!Context))" represents the lexical object in this parsing stage. This 
notation for lexical items has its origin in Lexical Functional Grammar [Kaplan \& Bresnan], so this expression can be represented like (†object) in the LIFG mannor.

This example states that if "ps" = [tal, then there should be honorific information in the resource situation of the individual who is the speaker If the speaker's RS contains two or moro different terms expressing the honorific relationship between the same agents, fail. 'Thus, the mechanism of 2) in dsolve is very important because it shows that in the honorific information of one individual there should not be different information about the binary honorific relation between two individuals.

(II) Second order honorifics Lexical rules for second order honorifies can be represented as in following example program.

Ex.4 This corresponds to $\mathrm{Bh}$ in 7) and 8) in Section 3.1 .2

$\operatorname{np}(\mathrm{X} 0, \mathrm{X} 2$, Contex $)<$

n(X0,X1,Context), sub3(X1,X2, Context)

dsolve(hono!((agent!(ds!Context))!(rs!Context), up(agent!(ds!X),obj!(lex!X))).

(III) Third order honorifics Lexical rules for third order honorifics can be represented as in the following example program

Ifx.5 'I'his corresponds to Eh in 10) in section 3.1 .2

$\mathrm{vp}(\mathrm{X} 0, \mathrm{X} 2$, Context) $<$

$\mathrm{v}(\mathrm{X} 0, \mathrm{X} 1, \mathrm{Context}), \operatorname{sub} 4(\mathrm{X} 1, \mathrm{X} 2$, Context),

dsolve(hono!((agent!(ds!Context))!(rs!Context),

up(agent! (lex!X), obj!(lex!X)))

3.2.3 Context $s$ witching in honorific sentences

When we utilize the contextual elements like DS and RS in discourse it is very difficult to decide the context for each sentence. A sentence in discourse can be represented by the expression "IDS,CS,I[alpha]]S,E", but then how do we map contexts like DS and CS to complox sentences?

Mizulani's theory of honorific forms does not go into context switching in a complex sentence. So we have expanded his grammar and propose a basic mechanism for context switching.

Consider sentence 1) below uttered by individual $S$ to $R$ which mean "individual 'I' said that individual U said that 'J'aro met Ilanako." In this example, we establish relations a) through j) among S, 'I', U, 'T'aro and llanako. Tho operater $>$ denotes the situation in which the left hand side honors the right hand side, < denotes the situation in which the right had side honors the left hand side, and $=$ denotes the situation in which there is no need to use honorifies between left hand side and right hand side.

The main point; in utterances of this form is that honorifies in these sentence change according to the form of speech, such as direct or indirect speech. But in the Japanese discourse there are no markot's like " " and "'I", so in order to process these sentences correctly, we need the mechanism of "context switching." Without this mechanism, all sentences would be parsed with one context, but this cannot explain the reason why honorifics change in complex sentences.

1) In S's utterance he said to R.

("I' said, U said, 'I'aro met Hanako")

"taro sama ga hanako sama ni ai nasat ta' to U ga iware ta' to 'I' ga iu ta

In RS of $\mathrm{S}$ a) $\mathrm{S}>$ Taro b) $\mathrm{S}>$ Hanako c) $\mathrm{S}>\mathrm{T}$ d) $\mathrm{S}>\mathrm{U}$ e) taro $>$ hanako

In $\mathrm{RS}$ of $\mathrm{T}$ f) $\mathrm{T}<\mathrm{U} \quad \mathrm{g}) \mathrm{T}^{\prime}=$ Taro $\quad$ h) $\mathrm{T}$ ' $<$ Hanako i) Taro $<$ IIanako

In $R S$ of $U$ j) $U<$ Taro k) $U<$ Hanakol) 'taro > Hanako

'These are the parsing rules used to analyze utterances.

L1) sentence -- $>\quad \operatorname{sn}(X)$, ds! $X=Y$, agent!Y $=s$, obj!Y $=\mathrm{r}$, at! $\mathrm{Y}=\mathrm{L}$

$f^{*} \mathrm{a}, \mathrm{b}, \mathrm{c}, \mathrm{d}, \mathrm{e}^{*} \mathrm{~J}$

hono! $(\mathrm{s} !(\mathrm{rs} ! X))=[$ down $(\mathrm{s}$, taro $)$, down $(\mathrm{s}$, hanako $)$ down(s,t), down(s,u), down(taro, hanako)],

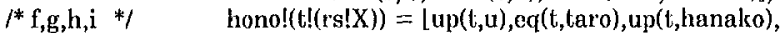
up(taro, hanako)l,

$f^{*} \mathrm{j}, \mathrm{k}, \mathrm{l} \quad$ */ hono!(u!(rs!X)) = [up(u,taro), up(u,hanako),

L2) $\operatorname{sn}(X) \rightarrow$

L3) $\operatorname{sn}(X) \rightarrow$ down(taro, hanako)].

$\operatorname{sno}(X),[$ to $], \operatorname{sno}(X)$

agent! (ds!Y) = agent!Z,ds!X = ds!Z,obj!(ds!Y) = obj!Z

L5) $\operatorname{sn}(\mathrm{X}) \rightarrow>\quad \operatorname{sn} 0(\mathrm{Y}),[$ to], sno(Z), agent!(ds!Y $)=$ agent!Z,ds!X $=\mathrm{ds} ! \mathrm{Z}$ obj! (ds!Y) = obj!Z

L1) and L2) are formal rules to start the process, while $(.3), L 4$ ) and 1.5 ) are basic rules for determining context switeh in sentences of discourse.

L1) specifies the inilial stage for parsing. In 1) above $S$ tells $R$ something so this context is set in the slot denoted by $\mathrm{ds} ! \mathrm{X}$.

L2) states that all of the features of sno are transfered to sn to meet a requirement of Mizutani's grammar. 'I'his is done easily by unification.

13) means that all of the features in sno are transfered to sn. 'This mechanism corresponds to indirect speech.

L4) means that there should be context switch. As the discourse situation for sn0, set agent of discourse of sno to agent of $Z$ who utters sno and set object of discourse of sn0 to object of $Z$ who hears this utterance.

L5) means that in a sentence with no marker, there can be context switch, so if a parsing failed because of the context of honorifics, use this rule.

Sontence 1) is analyzed using rule $[.5)$ and the mochanism of context switch is decrived from phase (I) to phase (III).

(l) Parsing really starts with the rule 3) estimating that there is no context switching. But at point *1, a conflict between $S$ 's resource situation and honorific expression occurs. In S's resource situation, the honorifie relation between $S$ and Taro is down(S,laro), but [taro, sama] requests the honorific relation up(S,taro), so context switch occurs at *2. Rule 5) switches the agent of discourse from $\mathrm{S}$ to $\mathrm{U}$. Context swilch does not occur at this point again. We use the notation I)S(S) to state that the agent of discourse is $\mathbf{S}$. The symbol $\rightarrow>$ means the context of left hand side is changed to the context of the right hand side as the result of eontext switch.

('T said U said 'I'aro met Ilanako)

taro sama ga hanako sama ni ai nasat ta to U ga iware ta to 'I' ga iu ta.

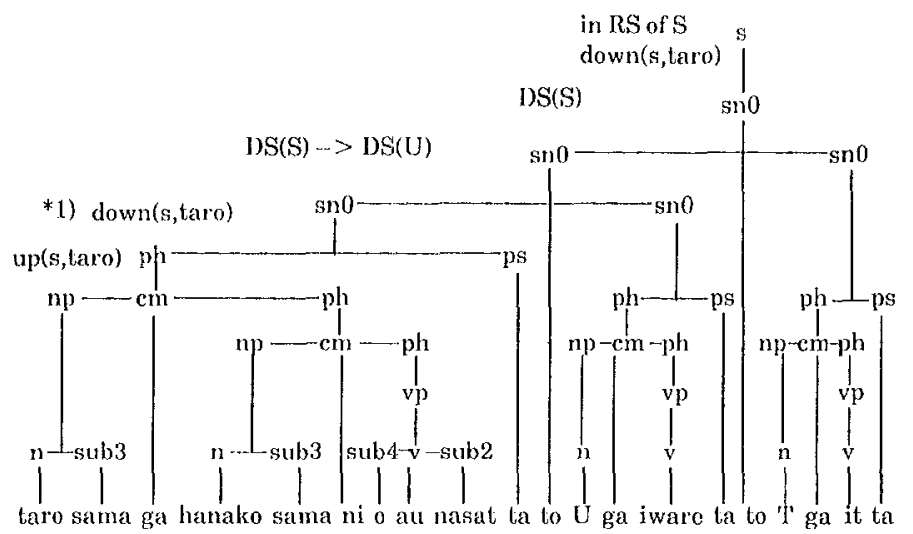

taro sama ga hanako sama ni o au nasat la lo $\mathrm{U}$ ga iware la to it ga it

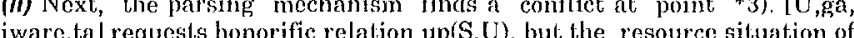
$\mathrm{S}$ contains the honorific relation down $(\mathrm{S}, \mathrm{U})$. 'Then context switch given in 5) is applied at point ${ }^{*} 4$ ).

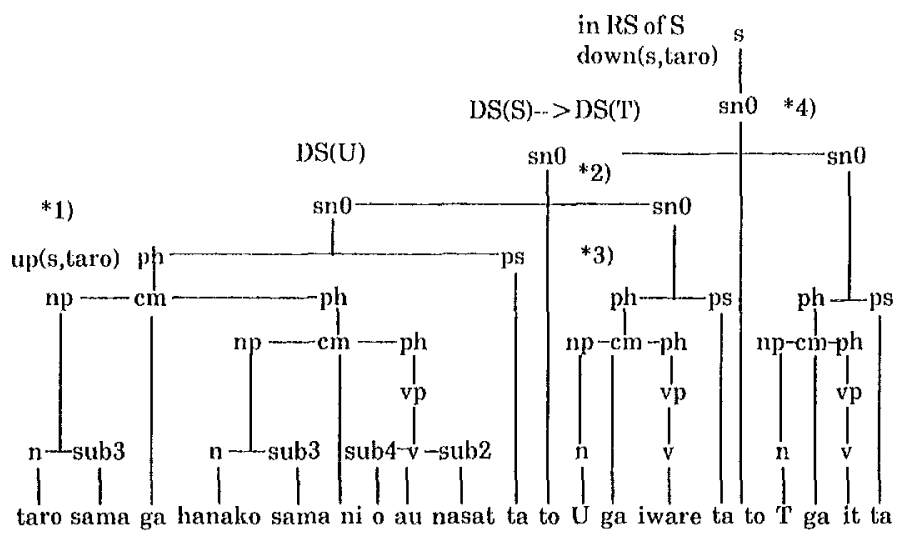

(III) Finally, this sentence is parsed like the following tree

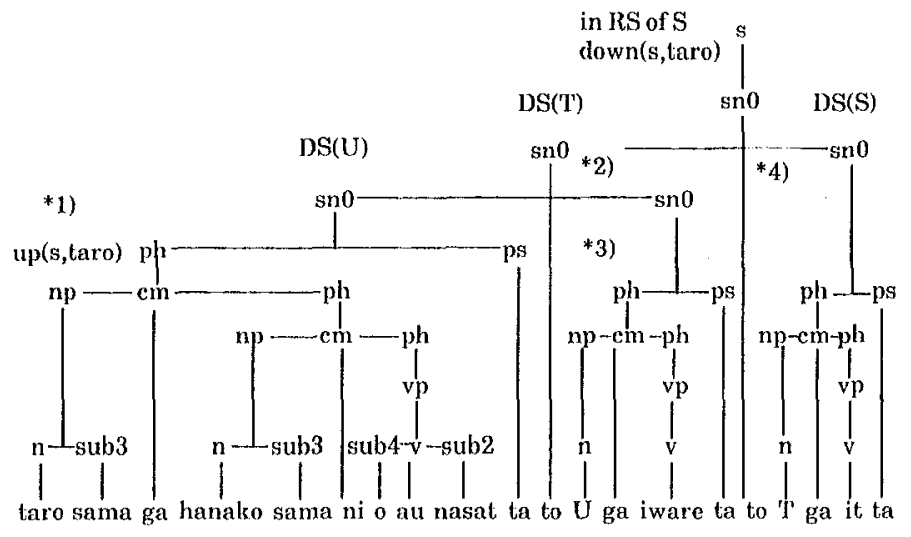

3.3. Ch and $\mathrm{Cw}$ 
Now, we have come to the main point of our model, but there remains an interesting feature of $\mathrm{Cw}$. 'This constraint is not verified so with some trepidation we touch on it briefly here.

For example, when a worker "Suzuki" refers to bis friend "Tanaka" with contempt, he will intentionally use a polite word to refer to him such as "Tanaka sensei" (Mr.Tanaka). When the hearer (Y)hears this polite expression, he decides on honorific event types but finds conflicts between these types and the normal social event types in his resource situation.

Ex.6

expression [Tanaka sensei] where Eh:= at l: honor up,Suzuki,Tanaka

expression [Tanaka sensei] where $\mathrm{Eh}:=$ at 1 : honor down,Suzuki,'Tanaka

in RS of $\mathrm{Y}$

HE : = Hr Y,Eh;yes

Eh := at lu: honor eq,'I'anaka,Suzuki

$\rightarrow$ hearer find conflict and the hearer wonders why !

Then the hearer (Y) wonders why he broke the universal honorific event. Finally, he comes to the conclusion that Suzuki intends either to praise Tanaka or berate him. We can go no further on this problem here.

There are other aspects to Wh. If the sentence is given first, wh will be calculated for each word and there remains a possibility of conflict betwoen honorific event types Fh in a simple sentence. In a complex sentence the mechanism of context switch will be used, but in a simple sentence this mechanism is not effective. When the hearer tries to deal with this conflict, he or she will assume that the speaker has some illegal honorific constraint Ch'. We have implemented this mechanism in our model system. 4. System Configuration

Our experimental system written in CIL runs on the DEC 2060 and utilizes Prolog as the basic programming environment, which enables us to use CIL. CIL is now compiled and runs very fast on DFC 2060. Next, we want to run this IDCG parser on the Bottom Up Jarser [Matsumoto 1983], [Matsumoto, Kiyono, 1984].

5. Some Other Examples

In this section, we give some examples which have no relation to context switching

\section{1) Sentence without honorific expression.}

A sentence without honorifics is parsed. 'I'hese are resource situations for this type of sentence.

|?- parse([laro,ga, hanako,ni,at, la],[],Context)

$\mathrm{RS}$

I_1855 :: anchor: $=1857$, relation: $=$ rs,agent: $==, \mathrm{r}$,

has: $\left.=[1626, \ldots 3422] \_3885\right]$

[ $1624::$ anchor: $=1 \overrightarrow{626}$, relation: $=$ honor, spoc: $=$ equi agent1: $=\mathrm{s}$, agent $2:=$ taro

3420:: anchor: $=\ldots 3422$, relation: $=$ honor, spec: $=$ equi agent: $=$ s, agent $2:=$ hanako ] ]

2) Sentence with illegal honorific expression

The following is an sentence with conflict between honorific word expressions. [taro] is a word withour honorifics but [o, ai,nasat] are words with honorifics from the speaker to 'Iaro. In a simple sentence, there should not be conflict between honorific relations. If there is, then the hearer $\mathbf{R}$ gets information that the speaker $\mathbf{S}$ has some trouble with honorific word expression.

|?- parse(DS,RS,CS, [taro,ga, hanako,ni,o, ai,nasat,ta],[]). RS.....

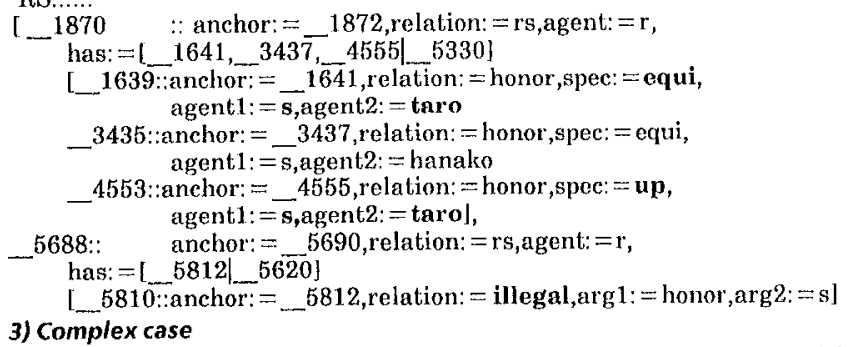

This example sentence contains many honorific expressions. The system analyzes these expressions to find some honorific event type in the speaker's mind.

[?- parse(DS,RS,CS,[taro,sama,ga,hanako,ni,o,ai,nasai,masita],[1). RS.

I $2545 \quad::$ anchor: $=2547$, relation: $=$ rs, agent: $=r$,

has: $=[2316,404 \overrightarrow{6}, 6558 \mid 7153]$

I $2314:$ anchor: $=-2316$, relation: $=$ honor,spec: $=$ up,

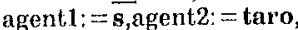

4044::anchor: $=4046$, relation: $=$ honor, spec: $=$ equi, agent1: $=\mathbf{s}$,agent $2:=$ hanako,

6556::anchor: $=6558$, relation: $=$ honor, spec: $=$ up agent $1:=\mathbf{s}$,agent $2:=\mathbf{r}] \quad 12068]$

\section{Conclusion}

It is easy to model honorific context in situation semantics. But we don't know how this context is represented in the human mind. This requires further research.

This treatment of the contexl switching mechanisms of honorifics is a first step toward analyzing more complicated phenomena. The main contribution of this model derives from the fact that in any complex sentence, there will probably be context switehing on honorifics. But this model shows context switching in a complex sentence only and there remains more complicated phenomena like the following

$S$ Hanako sama ni atta' [Hanako sama] is a honorific form (1 met Hanako.)

R 'Ianako sama tte dare?' (Who is "Hanako"?)

$|\ldots 1 \ldots|$

S 'Tanaka Hanako' (Hanako'Tanaka)

$R$ ' $\Lambda a$, hana no yatsu ka' [hana] is nonhonorific form.

(Oh, Hana!)

$\$ 1$ is the direct speech act and there should be a context switch because when $R$ knows who Hanako is, he refers to her with the nonhonorific "Hana." But we do not formalize the context switch which decides who is the agent of sentence $\$ 1$. 'T'o solve this problem, we should use an "anaphora mechanism for the honorific context" and in order to build a firm model of this mechanism, study not only of the anaphora mechanism [Barwise 1985c] but also the focusing mechanism [Sidner] is required. 'J'hese also are topic for further research

\section{ACKNOWLEDGEMEN'S}

1 would like to thank Mr. Mukai of ICO'T for his comments on context switching and Mr.Yokot, the llead of the Second Research Laboratory at ICO'l, for his encouragement and for allowing me ample time for this work.

\section{REFERENCW}

[Barwise 1982] Barwise, J., "Some Computational Aspects of Situation Semantics", $\Lambda C L(1982)$,pp. 109-111.

[Barwise 1984a] Barwise, J., "Lectures on Situation Semantics". lecture Notes for Winter Quartor(1984).

[Barwise 1984b| Barwise, J., "T'he Situation in Logic -- I", CSLI Report No. CSLI-84-2, March (1984)

[Barwise 1985a] Barwise, J., "The Situation in Jogic -- II", CSLI Report No. CSII-85-12, January (1985)

[Barwise 1985b] Barwise, J., "The Situation in Logic -.. III : Situation, Sets and the $\Lambda$ xiom of Foundation", CSLJ lReport No. CSIJI-84-26, June (1985).

[Barwise 1985e] l3arwise, J., "A Model of the Treatment of Anaphora in Situation Semantics", Informal Notes, No. IN-CSI.I-85-1(1985).

[Barwise 1985d] Barwise, J., "Notes on Situation 'Theory and Situation Semantics for CSLI Summer School", Notes for lectures duly 8-12(1985).

[Barwise 1985el Barwise, J., "On the Model 'Theory of Shared Information", Jeccure Note for July 16 (1985)

[Barwise \& Perry 1983] Barwise, J. \& Perry, J., Situations and Attitudes (MI'l' Press, 1983).

[Mizutani 1983a] S. Mizutani "Koku_bunpo so-byo (Sketch of Japanese Grammar)", Bunpo to imi (Grammar and Meaning), (Asakura 1983),pp.1-80 [Mizutani 1983b] S.Mizutani "'laiłu hyogen no shikumi (Structure of Honorific Expressions)", Unyo 1 (Pragmalics 1),( Asakuxa 1983), pp.158-178 [lesperance] Lesperance, Y., "l'oward a Computational Interpretations of Situation Semantics", (draft)

[Pollard 1985] Pollard, C.,e., "I'oward an Anadic Situation Semantics", (draft) (1985)

[Creary \& Pollard] Creary L.G., \& Pollard, C.J. "A Computational Semantics for Natural I anguage" (1985)

[Cohen] Cohen, P.,R., \& Levesque, H.,J., "Speech Acts and Rationality" ACI. (1985)

[Kaplan \& Bresnan] Kaplan, R.,M., \& Bresnan, J., "Lexical-Functional Grammar: A Formal System for Grammatical Representation"

[Mukai 1985a] K. Mukai "Ilorn Clause Logic with Parameterized 'I'ypes for Situation Semantics Programming" ICO'T' Technical Report No.'TR101, February, (1985)

Mukai 1985b] K Mukai "Unification over Complex Indeterminates in Prolog" ICO'] 'T'echnical Report No.'I'R1 13,June,(1985)

[Bowen 1982] D. Bowen (ed.), L.M.Byrd,F.C.N. Pereira, I.M.Pereira \& I] H.D.Warren "DECsystem-10 Prolog User's Manual,Department of Artificial Intelligence, University of Edingburgh,(1982)

[Sidner] Candace L.Sidner, "Focusing in the comprehension of definite anaphora" M. Brandy \& C. Berwick (ed.) Computational Models of Discourse (MIT Press 1983) pp.107-164

[Malsumoto 1983| Matsumoto, Y., et al., "BUP: A Bottom-Up Parser Embedded in Prolog," New Generation Computing, vol.1, no.2, pp.145-158, 1983.

[Matsumoto,Kiyono 1984] Matsumoto, X., Kiyono, M. and Tanaka, H, "Facilities of the BUP Parsing System," Proceedings of the 1st International Workshop on Natural Language Understanding and logic Programming, Rennes, France, Sept. 1984.\$ 